\title{
The potentially harmful excipients in prescribed medications in a Neonatal Intensive Care Unit in Kosovo and available safer alternatives
}

\author{
Blerina Koshi ${ }^{1,2}$, Arlinda Daka Grapci ${ }^{1 \oplus}$, Dashnor Nebija ${ }^{1 \oplus,}$ \\ Pranvera Breznica Selmani ${ }^{1 \oplus}$, Rina Morina ${ }^{1 \oplus}$, Maja Simonoska Crcarevska $^{2 \oplus}$, \\ Marija Glavas Dodov ${ }^{2 \oplus}$, Renata Slaveska Raichki ${ }^{2 \oplus}$ \\ ${ }^{1}$ Department of Pharmacy, Faculty of Medicine, University of Prishtina "Hasan Prishtina", Prishtina, Republic of Kosovo; ${ }^{2}$ Department \\ of Pharmaceutical Technology, Faculty of Pharmacy, University of Skopje "St. Cyril and Methodius", Skopje, Republic of North \\ Macedonia.
}

\begin{abstract}
Background. Medicinal products contain excipients that might be associated with toxicity in neonates. The aim of this study was to investigate the administration of medication containing potentially harmful excipients to neonates hospitalized in Kosovo and to identify the possibility of reducing neonatal exposure to these excipients through product substitution.

Methods. Data on all medication administered to hospitalized neonates from 1st of February to 1st of August 2018 along with patients' demographic data were collected from medical records for each neonate. Excipients were identified from the Summaries of Product Characteristics. Three stage criteria for product substitution were: (1) same active pharmaceutical ingredient (API) and route of administration; (2) 1 plus same dosage form; (3) 1 and 2 plus same strength.

Results. In total, 100 excipients were found in 2388 prescriptions comprising 67 medications and 60 API administered to 294 (183 preterm and 111 term) hospitalized neonates. The excipients of interest (EOI) were present in $409(17.1 \%)$ prescriptions and were administered to $131(71.6 \%)$ preterm and $52(46.8 \%)$ term neonates through a relatively small number of products $(\mathrm{n}=27 ; 32.8 \%)$. In relation to prescription frequency, the most common EOI was polysorbate 80, found in $229(56 \%)$ of EOI-containing prescriptions. Substitution with EOIfree products was possible for $14(63.6 \%), 12(54.5 \%)$ and $5(22.7 \%)$ products, according to the first-, second- and third-stage criteria, respectively.

Conclusions. We have provided the first detailed description of neonatal exposure to potentially harmful excipients among neonates admitted to a neonatal intensive care unit in Kosovo. Unnecessary exposure could be reduced by using EOI-free products available in the local medicine market. Collaborative initiative is required to build up the evidence on the use of EOI in neonates and raising awareness among health care professionals on use of products without EOI where possible.
\end{abstract}

Key words: neonates, medication, excipients of interest, neonatal intensive care unit, substitution.

Pharmaceutical excipients are important chemical constituents of medications to overcome challenges such as solubility, stability

$凶$ Arlinda Daka Grapci

arlinda.daka@uni-pr.edu

Received 27th July 2021, revised 6th September 2021, accepted 17th September 2021 and bioavailability of the active pharmaceutical ingredient (API). They also play a critical role in formulating, assuring the quality and patient acceptability of the medicine. ${ }^{1-4}$ The strategy for the selection of excipients is a complicated task in pediatric medicine development. It requires various considerations such as acceptable taste, age, dosage forms, among others to be accounted for in order to select safe excipients. ${ }^{5}$ Concerns 
about their safety are being more evident due to the increasing number of reports and awareness on adverse effects, especially in neonates. ${ }^{6}$ Adverse effects are prominent in neonates and primarily preterm neonates because due to their physiological and developmental immaturity they may not be able to handle an excipient in the same way as adults. ${ }^{7}$ In fact, tragedies have occurred after the inclusion of certain excipients in products used in neonates. ${ }^{8}$ Vulnerability of the neonates to excipients has been addressed in the European Medicines Agency (EMA) guideline on excipients that states "excipients to be used in formulations for the pediatric population should be selected with special care, and possible sensitivities of the different age groups should be taken into consideration".,79 Moreover, there is a new labelling guidance that should bring quantitative data about excipients to the Summary of Product Characteristics (SPC) and patient information leaflet (PIL) for new formulations. ${ }^{10}$ However, age-appropriate and excipient-low neonatal formulations are still scarce. Consequently, neonates may be at risk of relevant excipient exposure causing clinical harm. ${ }^{7}$

In order to enable researchers to more easily review important excipients, a list containing "excipients of interest" (EOI) has been proposed. ${ }^{11}$ Unfortunately, the use of these potentially harmful excipients in medicine administered to neonates is not a rare case in practice, as demonstrated in some previous studies. ${ }^{12-17}$ However, this issue remains largely unknown. On the other hand, according to a previous study ${ }^{18}$, exposure of neonates to these EOI could be reduced by using EOI-free formulations.

We are not aware of any study relating to EOI exposure among neonates in a European country that is not included in two previous European studies $^{15,18}$ on these topics. Given the need to reduce the prescription of EOI containing medications in the neonatal population and the lack of such studies in Kosovo, we aimed to investigate, based on a previous study ${ }^{17}$, the exposure to EOI among neonates admitted to a neonatal intensive care unit (NICU) in Kosovo and to identify substitution possibilities for medication containing these EOI.

\section{Material and Methods}

\section{Subjects and study design}

This retrospective study was conducted at the NICU, Department of Neonatology, University Clinical Centre of Kosovo (UCCK) from 1st of February to 1st of August 2018.

The following data were extracted from medical records for each hospitalized neonate (postnatal age $\leq 28$ days) in the NICU during the period of this study: demographic data (gestational age, gender, birth weight, date of birth), admission and discharge days, and data concerning each prescribed medication (international non-proprietary name, trade name, pharmaceutical dosage form, strength and route of administration). Data regarding prescriptions for glucose and electrolyte solutions, vaccines and blood products were excluded from the study.

Neonates were categorized based on their gestational age (GA) as extremely preterm $(<28$ weeks), very preterm ( 28 to $<32$ weeks), late preterm $(<37$ weeks) and term neonates $(\geq 37$ weeks $).{ }^{19}$

\section{Identification of excipients in prescribed medications for hospitalized neonates}

The excipient content of each prescribed medicine was identified from the SPC and/or manufacturers' websites. Our research, based on previous studies ${ }^{15-17}$, was focused on the EOI recognized as potentially harmful to neonates, namely benzalkonium chloride, benzyl alcohol, benzoic acid, sodium benzoate, ethyl-, methyl-, and propylparaben, ethanol, propylene glycol, 
polysorbate 80 saccharin sodium and sorbitol. ${ }^{15}$

\section{Analysis of the possibility of products' substitution}

Criteria for product substitution proposed by Nellis et al. ${ }^{18}$ and applied in our study were: stage 1: Medication could be substituted with a product with the same API and route of administration; stage 2: Stage 1 plus the requirement of the identical dosage form; stage 3: Stages 1 and 2 plus the requirement of identical strength of the API. For the analysis of the possibility of products' substitution, a search on the Kosovo Medicines Agency's (KMA) database on the list of products available locally ${ }^{20}$ was conducted by using the name of each API. Only EOI-free products were considered for substitution in the analysis.

\section{Statistical analysis}

Data were stored in Excel for Windows version 10 and analyzed in the Statistical Package for the Social Sciences version 20.0. Patients' data were analyzed using descriptive statistical methods (percentage proportion, mean and standard deviation [SD]). Nominal data were described as the quantity (n) and percentage with a 95\% confidence interval [CI]. Categorical data were analyzed with $\chi 2$ test $(2 \times 2$ tables). Data with $p$ value $<0.05$ were regarded as statistically significant. Categorical data were also described as the quantity and percentage proportion.

The study was approved by the ProfessionalEthics Committee of the University Clinical Center of Kosovo, under Protocol No. 853/2019. As we did not record personal identifying data for the neonates and no intervention was performed on patients, we did not seek consent for participation in the study.

\section{Results}

\section{Characteristics of the neonates and prescribed formulations}

During the study period, 299 neonates were admitted to the NICU, among whom $5(1.7 \%)$ with incomplete data were excluded. The 294 hospitalized neonates (183 (62.2\%) preterm) included in the study received 2388 prescriptions for 67 different medications and 60 different API (Table I). Intravenous formulations were used most often $(35 / 67 ; 52.2 \%)$, followed by oral formulations $(18 / 67 ; 26.9 \%)$ of which 10 $(55.6 \%)$ were oral solids such as manipulated tablets, 7 (38.9\%) oral liquids and 1 (5.6\%) oral gel. Inhalation $(5 ; 7.5 \%)$, ophthalmic $(4 ; 6.0 \%)$, topical $(2 ; 3.0 \%)$; intramuscular $(1 ; 1.5 \%)$, rectal $(1 ; 1.5 \%)$ and subcutaneous $(1 ; 1.5 \%)$ medication were scarcely used.

Table I. Demographic characteristics of neonates admitted to the NICU.

\begin{tabular}{lcccc}
\hline & Extremely preterm & Very preterm & Late preterm & Term \\
\cline { 2 - 5 } Characteristics & $\mathrm{n}(\%)$ & $\mathrm{n}(\%)$ & $\mathrm{n}(\%)$ & $\mathrm{n}(\%)$ \\
& $(95 \% \mathrm{CI})$ & $(95 \% \mathrm{CI})$ & $(95 \% \mathrm{CI})$ & $(95 \% \mathrm{CI})$ \\
\hline Neonates & $17(5.8)$ & $45(15.3)$ & $121(41.1)$ & $111(37.7)$ \\
Gestational age, weeks & $(3.1$ to 8.5$)$ & $(11.2$ to 19.4$)$ & $(35.5$ to 46.8$)$ & $(32.2$ to 43.2$)$ \\
(mean \pm SD) & $26 \pm 1.5$ & $30 \pm 0.8$ & $34 \pm 1.4$ & $39 \pm 1.3$ \\
Birth weight $(\mathrm{g})$ & $(22$ to 27$)$ & $(29$ to 31$)$ & $(32$ to 36$)$ & $(37$ to 42$)$ \\
Gender & $966(450$ to 1370$)$ & $1555(980$ to 4600$)$ & $2030(1200$ to 3150$)$ & $3395(2200$ to 5000$)$ \\
Female & & & & $40(36.0)$ \\
Female receiving EOI & $11(64.7)$ & $17(37.8)$ & $19(38.8)$ & $16(40.0)$ \\
Male & $7(63.6)$ & $16(94.1)$ & $72(59.5)$ & $71(64.0)$ \\
Male receiving EOI & $6(35.3)$ & $28(62.2)$ & $59(81.9)$ & $36(50.7)$
\end{tabular}

EOI: excipients of interest 


\section{The extent and nature of EOI administration}

Information on excipients was available for all 67 medications. The total number of excipients in all the studied medications was 100. Almost three-quarters of preterm $(131 / 183 ; 71.6 \%)$ and one-half of term $(52 / 111 ; 46.8 \%)$ neonates were exposed to at least one EOI. In relation to GA, very preterm neonates were most frequently exposed; in this subgroup, almost all (43/45; $95.6 \%$ ) neonates received at least one EOI. There was a difference between genders in the neonates' subgroups exposed or not exposed to EOI, but this difference was statistically significant only in the late preterm subgroup ( $\chi 2$ test, $p<0.05$; (Table I).

EOI were present in 409 (17.1\%) prescriptions. Of prescriptions, $81.6 \%$ (1949) were parenteral, however only $107(5.5 \%)$ contained EOI. In relation to the route of administration and frequency of prescriptions containing EOI, oral prescriptions were the most common (243/409; $59.4 \%$ ) (Table II).

Overall, 22 of $67 \quad(32.8 \%)$ medications contained at least one EOI, $50 \%$ of these medications (11/22) contained more than one
EOI. Intravenous formulations were most frequently used, but only $16.7 \%(6 / 36)$ of these formulations contained EOI. The proportion was higher in ophthalmic, oral, inhalation and topical medications: $25.0 \%(1 / 4), 55.6 \%(10 / 18)$, $60.0 \%(3 / 5)$ and $100 \%(2 / 2)$, respectively (Table III). Antimicrobial preservatives were found in 15 products, solvents in 8 products, sweetening agents in 7 products and solubilizing agents in 6 products. Concerning the specified excipient, methylparaben, found in 8 products, was the most common EOI, but it was present in only $16.6 \%(68 / 409)$ of prescriptions that contained EOI. In relation to prescription frequency, the most common EOI was polysorbate 80, found in $56.0 \%(229 / 409)$ of EOI-containing prescriptions. None of the formulations contained ethylparaben (Table III).

\section{Analysis of the possibility of products' substitution}

Regarding the possibility of product substitution proposed by Nellis et al. ${ }^{18}$ when applying the first-stage criteria, the substitution of medications containing EOI with EOI-free counterparts in Kosovo was possible for 14/22

Table II. Number of prescriptions and EOI prescribed by route of administration according to gestational age category.

\begin{tabular}{|c|c|c|c|c|}
\hline \multirow{3}{*}{ Characteristics } & Extremely preterm & Very preterm & Late preterm & Term \\
\hline & $\mathrm{n}(\%)$ & $\mathrm{n}(\%)$ & $\mathrm{n}(\%)$ & $\mathrm{n}(\%)$ \\
\hline & $(95 \% \mathrm{CI})$ & $(95 \% \mathrm{CI})$ & $(95 \% \mathrm{CI})$ & $(95 \% \mathrm{CI})$ \\
\hline Prescriptions (total n) & 149 & 506 & 1027 & 706 \\
\hline $\begin{array}{l}\text { Prescriptions with EOI } \\
\text { (total } n)\end{array}$ & $19(12.8)$ & $94(18.6)$ & $182(17.7)$ & $114(16.1)$ \\
\hline Intravenous & $120(80.5)$ & $339(67.0)$ & $712(69.3)$ & $483(65.7)$ \\
\hline Intravenous with EOI & $12(10.0)$ & $18(5.3)$ & $39(5.5)$ & $38(5.0)$ \\
\hline Intramuscular & $17(11.4)$ & $45(8.9)$ & $121(11.8)$ & $111(15.7)$ \\
\hline Intramuscular with EOI & 0 & 0 & 0 & 0 \\
\hline Oral & $7(4.7)$ & $100(19.8)$ & $162(15.8)$ & $68(9.5)$ \\
\hline Oral with EOI & $4(57.1)$ & $64(64.0)$ & $123(75.9)$ & $52(76.1)$ \\
\hline Inhalation & $5(3.4)$ & $16(3.2)$ & $28(2.7)$ & $29(7.2)$ \\
\hline Inhalation with EOI & $3(60.0)$ & $11(68.8)$ & $18(64.3)$ & $20(72.5)$ \\
\hline Other & 0 & $6(1.2)$ & $4(0.4)$ & $15(2.1)$ \\
\hline Other with EOI & 0 & 1(16.7) & $2(0.5)$ & $4(26.7)$ \\
\hline
\end{tabular}

EOI: excipients of interest, $n$ : number of prescriptions, 95\% CI: 95\% confidence interval.

Other: topical, ophtalmic, rectal and subcutaneous prescriptions. 


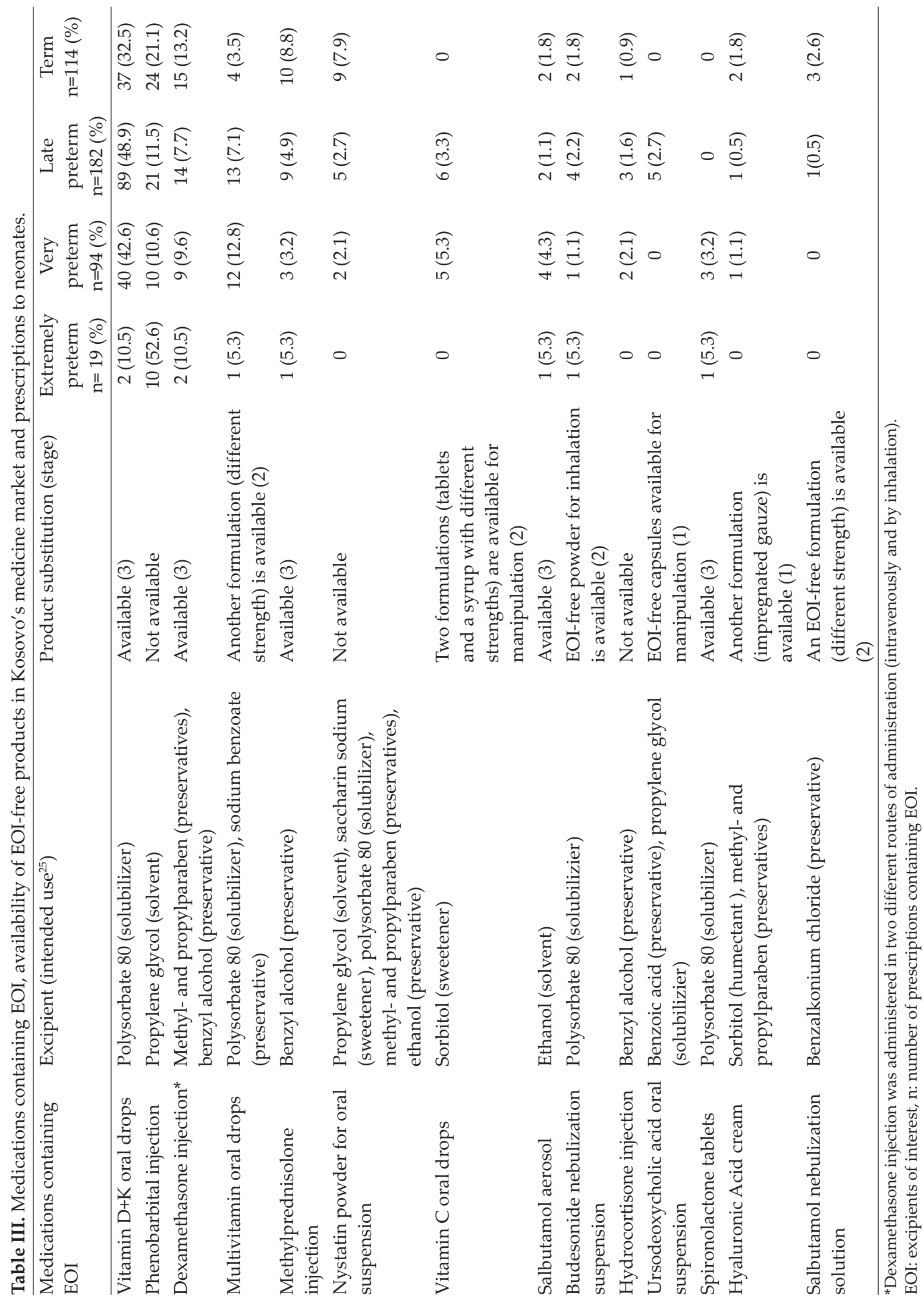




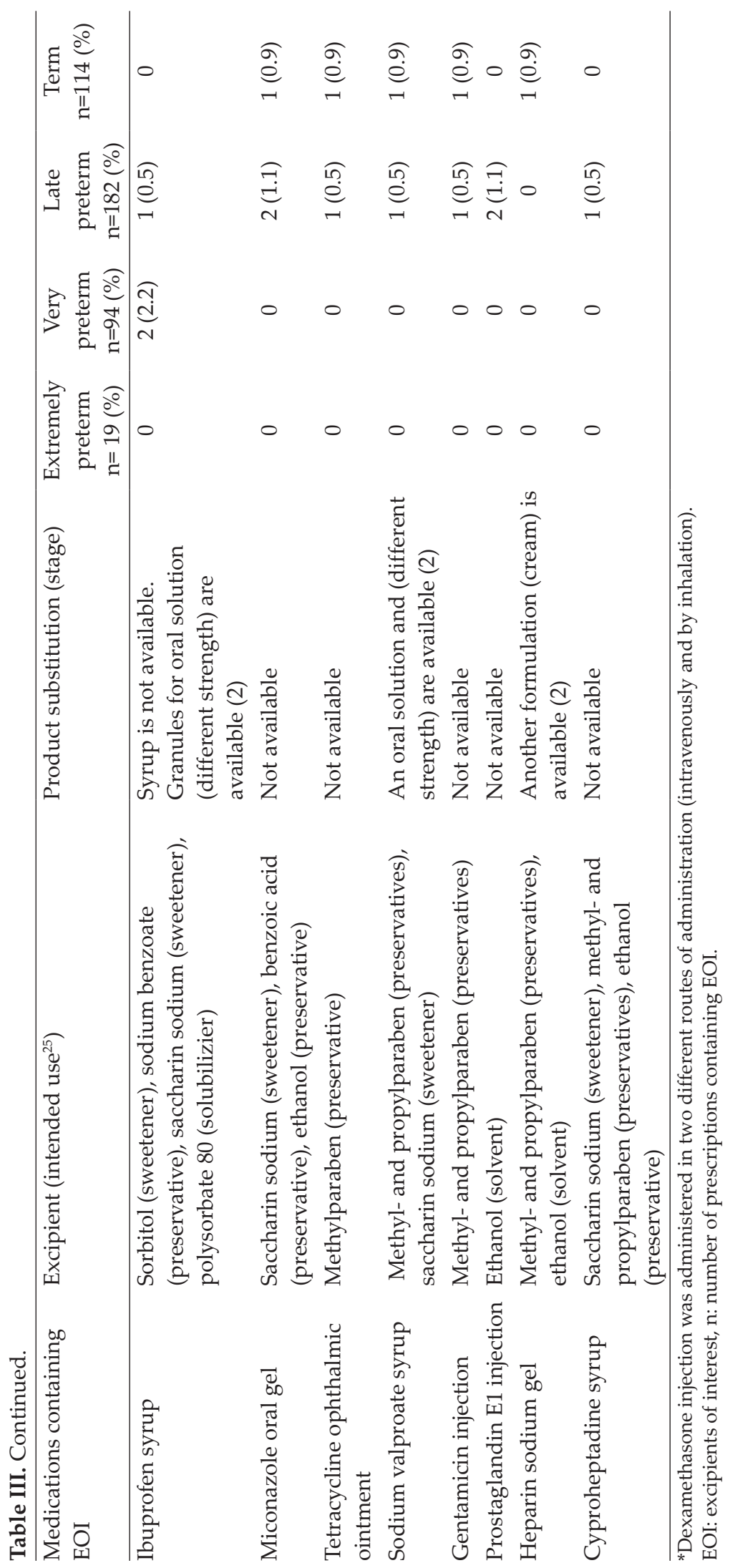


(63.6\%) products. By adding the second and third stage criteria, the possibility of product substitution was reduced to $12 / 22(54.5 \%)$ and $5 / 22(22.7 \%)$, respectively (TableIII). For example, ursodeoxycholic acid oral suspension, which contains benzoic acid and propylene glycol, could be substituted with manipulated EOIfree capsules (first-stage criteria). In addition, it is possible to substitute vitamin $\mathrm{C}$ oral drops (contain sorbitol) with vitamin $\mathrm{C}$ oral solution (second-stage criteria), and methylprednisolone injection $40 \mathrm{mg} / \mathrm{mL}$ (contains benzyl alcohol) with EOI-free methylprednisolone injection 40 $\mathrm{mg} / \mathrm{mL}$ (third-stage criteria) (Table III).

\section{Discussion}

Our study shows that while EOI were present in one-third of prescribed medications, approximately two-thirds of hospitalized neonates were exposed to at least one of these EOI. Generally, our data are in line with previous studies ${ }^{15-17}$, which also reported a small number of medications containing EOI, provided that in our study the frequency of exposure was higher, suggesting that a few, commonly prescribed medications cause a high frequency of EOI exposure and that substitution of this small portion of products containing EOI with EOI-free counterparts could spare a large number of neonates from exposure to these harmful excipients. ${ }^{15,16,18}$ Our findings on the opportunities for products' substitution make this suggestion even more reasonable; we identified a relatively high possibility of products' substitution and demonstrated that substitution of products that had alternatives with the same API as well as dosage forms would significantly reduce the exposure to EOIcontaining prescriptions.

The proportion of prescriptions containing at least one EOI in our study was the same as that reported by Saito et al. ${ }^{16}(17 \%)$, whereas studies performed by Nellis et al. ${ }^{15}$ and Sviestina and Mozgis ${ }^{17}$ reported higher percentages of these prescriptions (31\% and 28\%, respectively). We assume that differences in clinical settings protocols, as well as antibiotic prescribing practices, may account for this difference with the two latter studies. For example, gentamicin injection contains two EOI. In our study, there were only two prescriptions for gentamicin injection, while this medicine accounted for the largest number of EOI-containing prescriptions in the Latvian study. ${ }^{17}$

An important factor associated with EOI exposure was the route of administration. The main route of administration in hospitalized neonates is parenteral..$^{21}$ However, parenteral formulations may not require some kind of excipients, for example antimicrobials, as they can be produced in single dose vials. In our study, $84 \%$ of parenteral formulations and $77 \%$ of parenteral prescriptions did not contain EOI at all. These findings are similar to those reported by Sviestina and Mozgis ${ }^{17}$, who reported that only $13 \%$ of parenteral products and $17 \%$ of parenteral prescriptions contained EOI. Moreover, in the multi-country study, only $15 \%$ of parenteral prescriptions contained benzoates and parabens, suggesting that these excipients could be avoided. ${ }^{15}$

Polysorbate 80 was the most frequently present EOI in prescribed medications, even though it was found in a smaller number of products compared to methylparaben. Our results are similar to two previous studies looking at the specified EOI present in administered medications to neonates. Souza et al. ${ }^{13}$ reported higher exposure to polysorbate 80 compared to methylparaben ( $73 \%$ vs $57 \%$ of neonates). Similarly, Saito et al. ${ }^{16}$ demonstrated that while only $0.03 \%$ of neonates received parabens, $23 \%$ were exposed to polysorbate 80 . The main reason for high exposure to polysorbate 80 in our study could be its presence in vitamin $\mathrm{D}+\mathrm{K}$ preparation given to $57 \%$ of neonates, which is used for daily prevention of late hemorrhagic disease and rickets to all neonates from the 8th day of life. Importantly, we demonstrated that this product could be substituted according to the "ideal" criteria with an EOI-free counterpart in the local market. Moreover, given that the use of this product continues after hospitalization, 
we suggest that the overall cumulative benefit would be greater. On the other hand, we found no substitution possibility for phenobarbital, the second most commonly used EOIcontaining medicine that exposed 68 neonates to propylene glycol, whereas EOI-free injectable phenobarbital formulations are available in the European market. ${ }^{18}$

Antimicrobial preservatives should be included in medications only if absolutely needed. ${ }^{9}$ Not surprisingly, the pharmaceutical industry is encouraged to consider and develop novel strategies that allow commercialization of preservative-free products since mainly liquid dosage forms are used in these vulnerable patients. In our study, $68 \%$ of medications contained these EOI. However, we also demonstrated that $60 \%$ of medications containing these excipients could be substituted with EOI-free alternatives. In our study, parenteral medications contained antimicrobials and solvents. Of the 4 parenteral formulations containing only antimicrobial preservatives, we found substitution opportunities for 2 of them, whereas substitution was not possible for parenteral prostaglandin E1 containing ethanol, suggesting that the availability of products free from EOI may be related to the role of specified excipient in a formulation. ${ }^{15,18}$ This could be explained by the fact that is easier to avoid preservatives like parabens in the manufacturing process of single-dose parenteral medications than avoiding a solvent like ethanol. The situation is even more complex in the case of oral multi-dose liquid formulations where excipients like sweetening agents are needed to improve consistency and palatability. ${ }^{18,21}$ Our results show that 6 oral medications $(60 \%)$ contained sorbitol and saccharin sodium. However, substitution was possible for two-thirds of them, whereas in the study performed by Sviestina and Mozgis ${ }^{17}$ no substitution possibilities for oral medications containing sweeteners were available.

As expected, the possibility of substitution was not the same for all products. For the majority of products, it would be necessary to ignore the requirement for an identical dosage form. The proportion of products containing EOI that could be substituted, according to three stage criteria (first, second and third, respectively), with EOI-free products in our study was lower than that reported by Nellis et al. ${ }^{18}$ (88\%, 66\% and $31 \%$, respectively), but higher than in the study performed by Sviestina and Mozgis ${ }^{17}$ (31\%, 24\% and 14\%, respectively). Regional characteristics (e.g., products marketed in each country) may have had an influence on these differences.

Excipients may be harmful not only to neonates, but also to other patient populations such as critically ill adults, and children. ${ }^{22}$ However, considering the greater vulnerability of neonates, especially critically ill ones, to the toxicity of drugs and excipients, and little is known about this subject, this research was focused on this particularly vulnerable patient population. Almost two-thirds of our study population consisted of preterm neonates and this further increases the importance of our findings. ${ }^{13,23,24}$ The possible difference in the number of patients exposed to at least one EOI between preterm and term neonates may be polypharmacy, comorbidity or length of hospital stay.

Considering our findings, which indicate that health care professionals (HCP) lack awareness about the safety of excipients, the focus should be on raising prescriber's awareness about the excipients present in medications used in neonates by offering information and education about EOI and integrating scientific evidence in the decision-making process. Another solutionoriented approach could be the development of alert systems that calculate the number and ratio of excipients in concomitant medications with a patient-centered approach and alert them through integrated Computerized Physician Order Entry (CPOE). In addition, when compiling a formulary for the treatment of neonates, attention should be paid to the identification of EOI in medications 
and substitution of these EOI-containing medications with EOI-free counterparts available in the local market. We agree with Nellis et al. ${ }^{18}$ that substitution may incur additional costs as different stakeholders such as financial, regulatory and hospital specialists are involved. However, considering the overall health benefit of this susceptible patient group, this should be considered a valuable approach in sparing many neonates from exposure to these potentially harmful excipients.

Some limitations should be considered. There was limited information about quantitative data on excipients (single and daily dose of the excipient). In addition, this study was conducted in a small country with a developing health care system, which can make comparison with the results of other countries more difficult.

We have provided the first detailed description of neonatal exposure to potentially harmful excipients among neonates admitted to a NICU in Kosovo. Neonates in our country are exposed to several EOI which might be associated with toxicity. This exposure could be prevented or reduced by using EOI-free products available in the local market. However, collaboration is required to build up the evidence on the use of EOI in neonates and to raise the awareness among HCP on the use of products without EOI where possible, in accordance with the best practice standards. Our findings also constitute an important contribution to the strengthening of the national regulatory environment for pediatric medications registration in Kosovo.

Further studies are required to determine a balance of risks which include potential toxicity, exposure to EOI and safe substitution of medications as well as cost considerations.

\section{Ethical approval}

The study was approved by the ProfessionalEthics Committee of the University Clinical Center of Kosovo, under Protocol No. 853/2019.

\section{Author contribution}

Study conception and design: BK, AG and RR; data collection: RM, BK and AG; analysis and interpretation of results: DN and PS; draft manuscript preparation MD, MC and RR. All authors reviewed the results and approved the final version of the manuscript.

\section{Source of funding}

The authors declare the study received no funding.

\section{Conflict of interest}

The authors declare that there is no conflict of interest.

\section{REFERENCES}

1. FabianoV, Mameli C, Zuccotti GV. Paediatric pharmacology: remember the excipients. Pharmacol Res 2011; 63: 362-365. https://doi.org/10.1016/j. phrs.2011.01.006

2. Shehab N, Lewis CL, Streetman DD, Donn SM. Exposure to the pharmaceutical excipients benzyl alcohol and propylene glycol among critically ill neonates. Pediatr Crit Care Med 2009; 10: 256-259. https://doi.org/10.1097/PCC.0b013e31819a383c

3. De Cock RF, Knibbe CA, Kulo A, et al. Developmental pharmacokinetics of propylene glycol in preterm and term neonates. Br J Clin Pharm 2013; 75: 162171. https://doi.org/10.1111/j.1365-2125.2012.04312.x

4. Marek E, Kraft WK. Ethanol pharmacokinetics in neonates and infants. Curr Ther Res Clin Exp 2014; 76: 90-97. https://doi.org/10.1016/j.curtheres.2014.09.002

5. Rouaz K, Chiclana-Rodríguez B, Nardi-Ricart A, et al. Excipients in the paediatric population: a review. Pharmaceutics 2021; 13: 387. https://doi.org/10.3390/ pharmaceutics 13030387

6. Cuzzolin L. Neonates exposed to excipients: concern about safety. J Pediatr Neonat Individ Med 2018; 7: e070112-e070112.

7. Valeur KS, Holst H, Allegaert K. Excipients in neonatal medicinal products: never prescribed, commonly administered. Pharm Med 2018; 32: 251258. https://doi.org/10.1007/s40290-018-0243-9 
8. Phelps DL. E-ferol: what happened and what now?. Pediatrics 1984; 74: 1114-1116. https://doi. org/10.1542/peds.74.6.1114

9. European Medicines Agency. Guideline on excipients in the dossier for application for marketing authorisation of a medicinal product. 2007. Available at: https://www.ema.europa.eu/en/documents/ scientific-guideline/guideline-excipients-dossierapplication-marketing-authorisation-medicinalproduct-revision-2_en.pdf (Accessed on December $5,2020)$.

10. Annex to the European Commission guideline on 'Excipients in the labelling and package leaflet of medicinal products for human use' (SANTE-2017-11668). Available at: https://www. ema.europa.eu/en/documents/scientific-guideline/ annex-european-commission-guideline-excipientslabelling-package-leaflet-medicinal-productshuman_en.pdf (Accessed on May 30, 2021).

11. Gupta A, Khan MA. Challenges of pediatric formulations: a FDA science perspective. Int J Pharm 2013; 457: 346-348. https://doi.org/10.1016/j. ijpharm.2013.08.064

12. Lass J, Naelapää K, Shah U, et al. Hospitalised neonates in Estonia commonly receive potentially harmful excipients. BMC Pediatr 2012; 12: 1-11. https://doi.org/10.1186/1471-2431-12-136

13. Souza A, Santos D, Fonseca S, et al. Toxic excipients in medications for neonates in Brazil. Eur J Pediatr 2014; 173: 935-945. https://doi.org/10.1007/s00431014-2272-z

14. Fister P, Urh S, Karner A, Krzan M, Paro-Panjan D. The prevalence and pattern of pharmaceutical and excipient exposure in a neonatal unit in Slovenia. J Matern Fetal Neonatal Med 2015; 28: 2053-2061. https://doi.org/10.3109/14767058.2014.976549

15. Nellis G, Metsvaht T, Varendi H, et al. Potentially harmful excipients in neonatal medicines: a pan-European observational study. Arch Dis Child 2015; 100: 694-699. https://doi.org/10.1136/ archdischild-2014-307793

16. Saito J, Akabane M, Ishikawa $Y$, Nakamura H, Yamatani A. Potentially harmful excipients in neonatal medications: an observational and crossregional comparison of Japan and Europe. Neonat Pediatr Med 2018; 4: 2. https://doi.org/10.4172/25724983.1000172
17. Sviestina I, Mozgis D. A retrospective and observational analysis of harmful excipients in medicines for hospitalised neonates in Latvia. Eur J Hosp Pharm 2018; 25: 176-182. https://doi. org/10.1136/ejhpharm-2016-001107

18. Nellis G, Metsvaht T, Varendi H, et al. Product substitution as a way forward in avoiding potentially harmful excipients in neonates. Pediatric Drugs 2016; 18: 221-230. https://doi.org/10.1007/s40272-0160173-5

19. World Health Organization. Preterm birth 2018 Available at: https://www.who.int/news-room/factsheets/detail/preterm-birth (Accessed on December 22, 2020).

20. Kosovo Medicines Agency. List of products with Marketing Authorization in the Republic of Kosovo. Available at: https://akppm. rks-gov.net/Documents AndPublications/ DocumentsAndPublications/1092 (Accessed on November 8, 2020).

21. European Medicines Agency. Guideline on pharmaceutical development of medicines for pediatric use. 2013. Available at: https://www. ema.europa.eu/en/documents/scientific-guideline/ guideline-pharmaceutical-development-medicinespaediatric-use_en.pdf (Accessed December 3, 2020).

22. Sridharan K, Hasan HM, Al Jufairi M, et al. Possible effects of excipients used in the parenteral drugs administered in critically ill adults, children, and neonates. Expert Opin Drug Saf. 2020; 19: 1625-1640. https://doi.org/10.1080/14740338.2020.1805431

23. Giacoia GP, Taylor-Zapata P, Zajicek A. Drug studies in newborns: a therapeutic imperative. Clin Perinatol 2012; 39: 11-23. https://doi.org/10.1016/j. clp.2011.12.016

24. Allegaert K, Langhendries JP, van den Anker JN. Educational paper: do we need neonatal clinical pharmacologists? Eur J Pediatr 2013; 172: 429-435. https://doi.org/10.1007/s00431-012-1734-4

25. Sheskey PJ, Hancock BC, Moss GP, Goldfarb DJ. Handbook of Pharmaceutical Excipients. Pharmaceutical Press, 2020. 